Territoires, mobilités et santé : comment composer et penser une offre de soins pour les populations migrantes en situation précaire?

\title{
Anne-Cécile Hoyez
}

\section{(2) OpenEdition}

Journals

Édition électronique

URL : http://journals.openedition.org/rfst/456

DOI : $10.4000 /$ rfst. 456

ISSN : 2492-3672

Éditeur

Espaces et SOciétés (UMR 6590)

Référence électronique

Anne-Cécile Hoyez, «Territoires, mobilités et santé : comment composer et penser une offre de soins pour les populations migrantes en situation précaire ? ", Revue francophone sur la santé et les territoires [En ligne], Miscellanées, mis en ligne le 13 mai 2015, consulté le 06 avril 2021. URL : http:// journals.openedition.org/rfst/456 ; DOI : https://doi.org/10.4000/rfst.456

Ce document a été généré automatiquement le 6 avril 2021.

La Revue francophone sur la santé et les territoires est mise à disposition selon les termes de la Licence Creative Commons Attribution - Pas d'Utilisation Commerciale - Partage dans les Mêmes Conditions 4.0 International. 


\title{
Territoires, mobilités et santé : comment composer et penser une offre de soins pour les populations migrantes en situation précaire?
}

\author{
Anne-Cécile Hoyez
}

1 Cet article vise à interroger la place des enjeux de santé relatifs aux populations migrantes primo-arrivantes en les interrogeant du point de vue de l'expérience des individus et des professionnels qui travaillent à leur contact ${ }^{1}$. Les constats et analyses qui ont été produits à ce sujet depuis la fin des années 1990 dans la recherche portent à s'interroger sur tout ce qui se joue sur le continuum des soins et sur tous les éléments relevant de l'expérience migratoire au quotidien. En effet, les travaux réalisés jusque là dans ce cadre soulignent que les migrants primo-arrivants sont, plus que les autres, sujets à rester (au pire) en marge du système de santé et/ou (au mieux) cantonnées à des canaux spécifiques de l'accès aux soins, et ce en raison de l'interférence avec les logiques des politiques d'immigration. L'article revient sur cet état de l'art et, de façon transversale, il interroge les déterminants sociaux de la santé et de l'accès aux soins en prenant en compte l'ensemble des contraintes, des ruptures, mais aussi des ressources mobilisables pour la santé et les soins. L'article rend compte du point de vue des populations migrantes et analyse en miroir ce que les professionnels de la santé mettent en place à leur égard, dans un contexte institutionnel changeant, que ce soit au sein des politiques de santé ou des politiques migratoires. En effet, comment, dans le contexte politique et institutionnel actuel, mettre en place des actions à destination de ces publics? Comment faire en sorte que les organismes et structures existantes s'adaptent aux situations des populations les plus susceptibles de rester en marge du système de santé ?

2 Tous ces éléments plaident pour une prise en compte de la dimension spatiale et territoriale des expériences des personnes et des modalités de mise en œuvre des politiques publiques en matière de santé et de social. En effet, dans la mise en œuvre 
d'actions à destination des migrants, on note que de nombreux objectifs répondent aux impératifs de territorialisation, à l'échelle de la ville (et de ses quartiers) de l'action publique en matière de santé, de social et d'immigration (Hassenteufel et al. 1998; Fassin 1998). Cependant, sur le terrain, il existe un vif contraste entre cette posture «territorialisante » de l'action publique et les besoins des populations et des acteurs du soin, où les réalités sont marquées par les mobilités - contraintes ou choisies - des migrants comme des professionnels. En effet, alors qu'on observe certaines difficultés pour les migrants à se déplacer dans la ville pour avoir accès aux lieux de soins, entre autres, ou certaines capacités à mobiliser des réseaux à distance pour favoriser l'accès à l'information médicale, on observe également des pratiques professionnelles marquées par les déplacements au plus près des populations (des pratiques de l'« aller vers »).

3 Pour illustrer cela, l'article reviendra de façon synthétique sur les contextes actuels régissant l'accès aux soins des migrants, puis proposera une réflexion sur la dialogique « territoires et mobilités » dans les recherches en mettant en miroir les expériences du point de vue des patients comme de celui des professionnels.

4 Cette contribution aura donc pour objectif de proposer quelques éléments de débats sur la place et la pertinence des territoires et des mobilités en santé au regard de la situation de populations spécifiques. Il s'agira de travailler ensemble la question des expériences des populations, en miroir de leur trajectoire migratoire, et des pratiques des professionnels, au prisme de l'organisation des acteurs de la santé et du social tel qu'il se dessine aujourd'hui.

\section{Rappels : les contextes de l'accès aux soins pour les primo-arrivantS}

5 Deux constats principaux ressortent de l'étude de l'accès aux soins des primo-arrivants. Tout d'abord, c'est surtout le retard dans les démarches de santé, voire le non-recours, qui caractérise les parcours de soins (Médecins du Monde, 2009; Mizrahi \& Mizrahi, 2000). Ensuite, les démarches de santé n'apparaissent vraiment « coordonnées » (entre structures spécifiques et celles dites de droit commun) que lorsque la situation administrative et résidentielle est stabilisée (Hoyez, 2012). De fait, les modalités d'accès aux soins sont complexes et, sur le terrain, on observe tant des pratiques de nonrecours ou de report des soins qu'une utilisation plus fréquente de centres médicaux pour publics précaires ou recours à des dispositifs qui leur sont spécifiquement dédiés, quand ils existent (Hoyez, 2011; Warin, 2013). Ainsi, en plus des barrières à l'accès aux soins communes à toutes les populations précaires, les primo-arrivants expérimentent des parcours de soins marqués par des contextes défavorables (Rode 2009). Les effets délétères des conditions de vie résident tout d'abord dans leur situation administrative : les changements de statuts (qui peuvent faire basculer les personnes dans les catégories dites de l'illégalité) dissuadent les patients de se rendre dans les organismes de santé (Cognet, Gabarro, et Adam-Vezina 2009). Ensuite, la situation par rapport au logement prend le pas dans le lot des préoccupations quotidiennes : il est pour beaucoup une condition primaire à satisfaire avant d'entamer toute autre démarche. Entrent également en ligne de compte les difficultés linguistiques qui ont pour conséquences d'entraver les démarches pour s'adresser à un établissement de santé où à un guichet de l'assurance maladie, et peuvent se répercuter sur la compréhension du diagnostic et des recommandations du médecin (puis de la 
pharmacie) (de Jonckheere et al. 2011 ; Marche et Pian 2015). Enfin, peuvent intervenir des barrières qui relèvent de la présence de représentations de l'altérité dans la maladie et la façon de la concevoir et de la dire - aussi bien du côté du patient que du soignant (Cognet et Montgomery 2007). L'existence de telles représentations peut avoir des conséquences importantes sur les démarches de santé ou leur report.

6 Ainsi, la question de l'accès aux soins primaires des primo-arrivants ne révèle qu'une toute petite partie des difficultés éprouvées dans l'expérience migratoire. Les entraves liées au statut administratif (notamment à l'obtention d'un titre de séjour stable) imposent des difficultés d'orientation dans le système de droit commun (accès à un logement, à un travail et à des droits à l'assurance maladie). Dans le domaine des droits à la santé, la mise en place de la CMU et de l'AME ont permis de réguler l'accès administratif aux soins pour les populations en difficulté socio-économique (CMU) ou les populations en situation irrégulière (AME); mais ils ont aussi contribué à la marginalisation et à la stigmatisation des personnes relevant de ces dispositifs (Maille \& Veïsse, 2000). Par conséquent, depuis le début des années 2000, les recherches en anthropologie et en sociologie de la santé ont mis en lumière l'émergence de représentations qui donnent lieu à des processus de discrimination à l'égard des populations migrantes : Estelle Carde décrit très précisément ces processus en termes de discriminations $(2006,2007)$ et Marguerite Cognet et Christelle Hamel (2012) ont pu en faire une analyse quantitative par suite de l'enquête TeO de l'INED (Beauchemin, Hamel, et Simon 2010). Ainsi, les difficultés repérées dans l'accès aux soins des populations migrantes en France nous renseignent surtout sur les expériences de la migration au quotidien (Cognet et al., 2012) qui sont marquées, principalement, par les effets délétères de l'instabilité résidentielle sur la santé et l'accès aux droits des personnes. Les trajectoires de soins des migrants sont donc marquées par un ensemble de barrières qui permettent de lire, au-delà des entraves "mécaniques " d'accès aux soins, le report de multiples difficultés quotidiennes sur l'expérience de la santé: comment se soigner lorsqu'on expérimente des mobilités contraintes dans la ville (instabilité résidentielle, devoir aller se procurer de la nourriture, des vêtements, des papiers dans des lieux dispersés) ? Comment mettre en œuvre des démarches de soins lorsque l'accès même aux droits nécessite de déclencher des recours auprès de guichets de différentes tutelles institutionnelles (santé publique, social, immigration), aux fonctionnements répondant à des impératifs différents? Si l'on sait que l'accès aux soins est réduit ou entravé pour toutes les populations précaires (Parizot 2003; Chauvin et Parizot 2005), on constate que, pour les migrants, les facteurs de retard ou de nonrecours se cumulent, et s'installent dans le temps en raison de toutes les contraintes du quotidien liées à leur statut de migrants. Le nombre d'obstacles relatifs aux contextes sociaux et politiques à prendre en compte est important, et les réponses des services de santé et du social encore éparses et fragiles; cependant elles existent et doivent être également étudiées.

7 Ces constats soulignent l'impérieuse nécessité de prendre aussi les dimensions sociospatiales de l'accès aux soins, notamment de se pencher sur la question des mobilités des populations migrantes et des professionnels travaillant auprès d'eux, et ceci dans et entre les lieux de soins et de vie, à l'échelle de la ville. Rappelons que, comme on le sait déjà, les mobilités liées à la santé ne se lisent pas uniquement dans l'accès à et la disponibilité de lieux de soins : elles doivent être abordées en prenant en compte les paysages locaux de l'accès aux soins (c'est-à-dire l'ensemble des structures 
spécifiques, des professionnels mobilisés, des logiques propres aux individus et/ou famille), mais aussi en incluant, comme cela est souligné par ailleurs (Vallée, Frohlich, Kestens, \& Shareck, 2014 ; Vallée, Roux, Chaix, Kestens, \& Chauvin, 2014), des données sur la possibilité, par les acteurs en présence, à s'approprier la ville et ses ressources au-delà des cadres territoriaux communs.

\section{La dialogique territoires / mobilités dans les travaux sur l'accès aux soins des migrants}

8 Comme nous venons de le voir, s'intéresser aux migrants primo-arrivants porte à souligner leur position particulière en raison des statuts et des droits qui leur sont accordés par la loi, et qui leur sont plus ou moins effectivement octroyés dans la réalité. Autrement dit, pour bien saisir toute la complexité de l'objet d'étude, il nous faut prendre en compte les contextes politiques nationaux et des différentes représentations qu'ils provoquent sur les migrants pour comprendre l'ensemble des constructions qui permettent ou empêchent l'accès des migrants aux ressources collectives (The Franco-British Working Group on Migration, Health and Wellbeing, 2009). Mais, en plus de cela, il est important de saisir aussi les processus à l'échelle locale ou micro, notamment en replaçant les parcours des migrants dans leur contexte quotidien (Demazière \& Samuel, 2010) : les dimensions biographiques des individus et de leur famille entre dans la réflexion et il s'avère nécessaire d'aller explorer ces effets contextuels et leurs incidences sur les trajectoires migratoires et expériences du quotidien (Lelièvre et Courgeau 1996). Dans le cas des recherches qui guident nos propos, la dimension individuelle et familiale a été prise en compte dans la méthodologie de recherche, tout autant que la dimension professionnelle dans leurs cadres politiques et leurs logiques d'action.

\section{Méthodologie de recherche}

9 Les éléments d'analyse qui donnent matière à cet article ont été recueillis au cours d'un programme de recherche qui avait pour objectif d'analyser les éléments déterminant l'accès aux soins des migrants primo-arrivants et leurs variations à l'échelle locale en étudiant et comparant différentes configurations locales ${ }^{2}$. Les recherches associaient deux entrées prioritaires: l'une centrée sur les dispositifs de soins en présence à l'échelle locale et leur dynamique de fonctionnement dans le temps et l'espace, et l'autre sur les personnes qui utilisent ou exercent dans ces lieux. L'approche socio-spatiale, qui fait l'identité scientifique du projet, a permis de travailler ensemble les éléments qui font rupture ou ressource dans la santé et le soin, $\mathrm{du}$ point de vue des patients comme des professionnels. Par conséquent, le projet s'intéressait aux trajectoires croisées d'individus entre expérience des soins, des migrations et postures des professionnels. En effet, l'approche via la notion de "trajectoire ", théorisée par A. Strauss et I. Baszanger (1991), permet d'aborder la question du travail effectué par les professionnels de la santé et/ou des patients (et leurs proches) dans les processus de soin, englobe leur caractère imprévisible, accorde une grande importance à la biographie, et porte attention aux conflits. La dimension spatiale de la notion de trajectoire a été particulièrement creusée. 
10 Sur le plan méthodologique, le projet se base sur des analyses de 20 entretiens ethnographiques; les grilles d'entretiens ont été construites par les chercheurs SHS de l'équipe et des professionnels de la santé (voir tableau 1).

Une vingtaine d'entretiens réalisés auprès de patients (16) et de professionnels (4) ont été mobilisés pour cet article. Ils ont été réalisés en Bretagne en 2013 : les personnes (patients et professionnels) ont été recrutées soit dans des lieux de soins localisés dans différentes villes bretonnes, soit dans des lieux de vie; certains ont été rencontrés plusieurs fois.

12 Cette méthodologie qualitative nous a permis de travailler avec un petit échantillon de personnes à l'échelle micro-locale, peu abordée dans les travaux sur les migrations et la santé. Par conséquent, le but recherché par ce type d'investigation n'est pas de "parler le langage de la représentativité » (Olivier de Sardan 1995): l'article n'a pas la prétention de représenter toutes les expériences de tous les primo-arrivants et de tous les médecins, mais vise plutôt, à travers la méthodologie de recherche adoptée, à rendre compte de diverses logiques d'action ou de registres de pratiques qui se jouent autour de l'accès aux soins, en termes de processus sociaux et spatiaux. Les éléments qui sont retranscrits ici ne reprennent pas tous les détails des entretiens, qui seront analysés par ailleurs; ils permettent, à ce stade, de mettre en avant ce qui fait la régularité des discours, des expériences et des actions que l'on peut observer à cette échelle d'analyse. C'est ainsi que l'article décrit et analyse les points de croisement entre expériences des soins et expériences migratoires, en incluant les expériences du point de vue de patients et les met en miroir avec celles des professionnels. Les premiers ont répercuté, lors d'entretiens qui duraient généralement 20 à 30 minutes, leur satisfaction vis-à-vis du système de santé et de l'accès aux soins mais ont surtout évoqué les effets délétères de l'expérience du quotidien sur leur état de santé et celui de leurs proches; l'article cherche à rendre compte de cela en s'appuyant sur quelques biographies synthétiques représentatives de ces processus. Les seconds ont fait ressortir, à travers des entretiens qui duraient au moins une heure, la prégnance de leur trajectoire professionnelle personnelle (leurs carrières ont pour point commun le passage par l'humanitaire) sur les modalités d'actions qu'ils mènent aujourd'hui, c'està-dire des actions originales que l'on pourrait qualifier de "déterritorialisées ", à destination les personnes en situation de précarité, ancrées dans une réflexion sur les capacités du système de santé, où la norme est la sédendarité, à intégrer tous les publics. 
Tableau 1 : Thèmes abordés dans l'entretien en fonction des acteurs interrogés.

\begin{tabular}{|c|c|}
\hline Entretiens & Thématiques abordées \\
\hline Patients & $\begin{array}{l}\text { - Trajectoires migratoires (situation socioéconomique dans le pays d'origine; } \\
\text { motivations et formes de circulations depuis le pays d'origine; trajectoires } \\
\text { résidentielles et/ou professionnelles) } \\
\text { - L'accès aux soins (pathologies éventuelles; les réseaux mobilisés pour l'accès aux } \\
\text { soins; les expériences de soins dans d'autres systèmes de santé ; relations à } \\
\text { l'information médicale; accès à des soins "alternatifs", pluralisme médical, } \\
\text { automédication ; rôle del's entourage ") } \\
\text { - Les perspectives individuelles et/ou familiales }\end{array}$ \\
\hline Professionnels & $\begin{array}{l}\text { - Profil de poste (missions, conditions de travail, carrière personnelle, conditions de } \\
\text { l'émergence de la question de la santé des migrants dans leur travail) } \\
\text { - La structure (historique de l'institution, son organisation et fonctionnement actuel, } \\
\text { le type de public concerné, les trajectoires de soins perçues de son point de vue) } \\
\text { - Les financements et partenariats (questions relatives aux collaborations } \\
\text { principales ou ponctuelles, relations aux tutelles administratives régionales ou } \\
\text { nationales, projets en collaboration, difficultés dans les collaborations) } \\
\text { - Les contextes politiques et juridiques (leurs évolutions, leurs effets (réformes dans } \\
\text { le champ de l'immigration, dans le domaine de la santé), mobilisations locales, } \\
\text { régionales, nationales) } \\
\text { Questions approfondies au sujet des carrières individuelles (situation familiale, } \\
\text { ldées pulltiques, situatiuns prưesslunnelles passées...) }\end{array}$ \\
\hline
\end{tabular}

\section{Le point de vue des patients}

13 L'idée d'un accès aux soins coordonné, logique, rationnel, facilité, inscrit dans le circuit des structures de soins dites de « droit commun » est généralement remise en question dans le cas de publics spécifiques comme les primo-arrivants (Hoyez, 2012) ou comme les publics en situation de précarité (Parizot, 2003; Warin, 2013). L'analyse des démarches de santé de ces personnes tend à montrer que les logiques sont plutôt individuelles et changeantes, en fonction des conditions de l'accueil, de l'arrivée et du paysage sanitaire et politique local (Chauvin et al. 2014).

entretiens menés auprès de migrants primo-arrivants en Bretagne permettent de mieux connaitre et comprendre comment les facteurs socioéconomiques classiques et les effets de contextes locaux se combinent et entrent en jeu dans l'accès aux soins des migrants. En dehors des entraves mentionnées plus haut, on se rend compte qu'il existe également trois sortes de ressources qu'ils peuvent mobiliser : structurelles, sociales ou spatiales. Les ressources structurelles concernent tout ce qui est de l'ordre du fonctionnement des dispositifs mis en place à destination des migrants (Izambert, 2010; Hoyez, 2011). Les ressources peuvent être également sociales, lorsqu'elles se rapportent aux actions d'un groupe constitué comme corps social (type réseau de compatriotes plus ou moins formel, associations) (Pian, 2012). Enfin, doivent être prises en compte les ressources dites spatiales, c'est-à-dire celles qui concernent l'accès à des savoirs, des pratiques, des personnes, multi-localisées (notamment dans l'organisation diasporique) et potentiellement mobilisables pour la santé (Ma Mung, 1999; Thomas, 2010). La dimension sociospatiale et réticulaire ne doit pas être omise dans ces 
réflexions (distances, mobilités, accès socio-spatial différencié et variable en fonction des lieux et des échelles d'analyse).

A l'échelle locale, tous les fondements des politiques régissant les actions à l'échelle internationale ou nationale sont remis en cause par les situations d'urgence vécues par les patients et les professionnels. Notamment, le principe fondamental d'universalisme est fortement remis en question, en raison de l'hétérogénéité des pratiques sur le terrain. C'est à cette échelle-là que l'on observe le nombre important de mobilisations ponctuelles, qui prennent forme notamment à travers la mise en place de structures de soins spécifiques pour les publics migrants. Les recours aux soins des migrants dans la ville sont jalonnés de lieux précis : les structures du système dit « de droit commun » et les structures d'accueil aux personnes exclues de ce même système (personnes en grande précarité). Pour ces dernières, il s'agit surtout de structures d'accueil résultant d'initiatives locales, aux statuts associatifs et aux missions reliées aux priorités repérées dans les politiques publiques locales (de la santé, ou de la ville), et aux objectifs constamment négociés entre acteurs locaux, et révisés à mesure des changements des orientations des politiques nationales (de santé, d'immigration, du social) (Hassenteufel et al., 1998 ; Hoyez, 2011). Le contexte local nous rappelle que les travaux qualitatifs qui sont réalisés à l'échelle micro traitent surtout de situations hétérogènes, remettent en question les notions de marginalité ou d'exclusion pour questionner la centralité de certains lieux (c'est ce que soulignent Djemila Zeneidi et Sébastien Fleuret quand ils parlent de personnes "fixes sans domicile» (2007) ou Céline Bergeon (2014) qui rappelle l'existence de lieux qui font ressource, même de façon éphémère, dans la mobilité).

Le cantonnement à des structures de soins spécifiques impose des mobilités spécifiques qui se superposent avec les mobilités du quotidien (accompagner un enfant à l'école, aller chercher de la nourriture, faire des démarches administratives...) et avec les temporalités des circulations et les mobilités à l'échelle de la vie d'une personne. Ceci peut avoir des conséquences importantes sur la santé psychologique des personnes : la contrainte spatiale oblige à mettre en place des tactiques pour se déplacer dans la ville et accéder aux différents services, comme cela apparait dans ces extraits biographiques synthétiques :

Natalia vient d'un pays d'Europe de l'Est. Elle est arrivée en France à 18 ans, après avoir circulé dans différents pays en quête de soins pour une pathologie hépatique mettant sa vie en danger. Prise en charge en CADA ${ }^{3}$, elle réside à $30 \mathrm{~km}$ de Rennes, dans un logement qu'elle partage avec d'autres personnes de nationalités différentes de la sienne. Sa pathologie nécessite un suivi régulier au $\mathrm{CHU}$ et dans un centre médical où officie une interprète ${ }^{4}$. Pour se rendre à Rennes, elle doit prendre un autocar qui ne passe qu'une fois par heure, et qui prend 40 minutes pour rejoindre le centre de Rennes. Elle doit ensuite prendre un métro ou un bus pour l'emmener dans son lieu de consultation. Dans la petite ville où elle réside, ses activités se résument à aller à l'église ou à la pharmacie. Elle ne connaît personne dans cette ville. Les quelques compatriotes qu'elle connaît sont à Rennes. Très satisfaite de la prise en charge médicale et d'un possible hébergement à Rennes (en cours de négociation, au moment de l'entretien, par l'équipe médicale pour lui permettre d'être mieux suivie). Se déplacer en dehors de sa commune de résidence est trop difficile à mettre en œuvre, elle préfère limiter ses déplacements longs à ses consultations, mais déclare néanmoins souffrir de l'isolement au quotidien.

Tariq vient du sous-continent indien. Il a fuit son pays avec sa femme et sa fille suite à 2 tentatives d'assassinat sur sa personne. D'abord pris en charge par un CADA en région parisienne, la famille a ensuite été déplacée dans l'agglomération 
rennaise. Ils habitent tous les trois dans une maison à la campagne, sans accès à pied à aucun service basique. Pour faire les courses, récupérer un paquet aux Restos du Cœur, du courrier à la Croix Rouge, des vêtements au Secours Populaire, ou bien aller consulter au centre médical ou en pédiatrie au CHU (leur enfant, en bas âge, est tombé plusieurs fois malade), ils doivent marcher 20 min jusqu'à l'arrêt d'un bus. Celui-ci passe toutes les heures entre $9 \mathrm{~h}$ et $17 \mathrm{~h}$, et prend 25 minutes pour rejoindre le centre de Rennes. Ils doivent ensuite prendre un bus ou métro pour se rendre dans les divers lieux où se procurer alimentation, vêtements, ou honorer une consultation médicale. Les parents sont très affectés de cette situation, si bien qu'en consultant pour leur enfant au CHU, l'équipe médicale a également mis en place un suivi psychiatrique : une équipe mobile vient leur rendre visite chez eux. Le couple est très satisfait du suivi médical, que ce soit pour leur enfant ou pour eux. Par contre, la situation d'isolement et le poids de tout déplacement sont pour eux une source d'angoisse importante.

17 Ces deux exemples, qui soulignent l'importante articulation entre parcours de soins, lieux de résidence et espaces de vie, mettent en avant la situation d'individus ou de familles fragilisées par une expérience du quotidien vécue comme angoissante. Cependant, ces situations sont à mettre en miroir avec les actions de différentes structures de soins qui tentent de prendre en compte le contexte résidentiel. En effet, pour certains professionnels, il est entendu que la garantie d'un accès aux soins correct et d'un suivi effectif passe par la stabilisation résidentielle dans un lieu où l'accessibilité à tous les autres services de base est possible (Yeni $2010^{5}$ ). C'est en cela que le Réseau Louis Guilloux à Rennes, à l'instar d'autres associations prenant en charge des patients atteints de maladies infectieuses, mettent en œuvre un ensemble d'actions visant à stabiliser la situation sociale des migrants qu'ils rencontrent en consultation. Si le point d'entrée est ici la santé, la palette des actions menées déclenche des réactions en chaîne dans le domaine du logement et des différentes formalités administratives.

\section{Le point de vue des praticiens}

18 Les recherches sur les pratiques des professionnels qui travaillent aux côtés de patients en situation de précarité soulignent la multiplication des actions relevant de «l'aller vers ». Cette pratique n'est pas vraiment nouvelle : elle est bien connue dans le milieu du travail médico-social, en particulier dans les actions de lutte contre les addictions (alcoologie, toxicomanie) ou contre le VIH-Sida. La conceptualisation de ce type de pratique est peu rencontrée dans le milieu académique car elle relève surtout du domaine du travail social, lui-même peu théorisé (Rullac 2014) ; c'est cependant une pratique de terrain qui se décline en différents types d'intervention visant à "aller vers » des publics identifiés comme précaires. Cette pratique sous-entend que les professionnels doivent se rendre là où se trouve la personne: dans la rue, mais aussi dans d'autres espaces (établissements scolaires, hôpitaux, centres sociaux, prisons...) (Roche, 2007). Maraudes, équipes mobiles médicales ou équipes de liaison interinstitutionnelles sont autant de dispositifs mis en place à cet effet. Sur le terrain, on rencontre beaucoup ce type de pratique dans les actions menées auprès des migrants primo-arrivants, qu'ils soient à la rue ou en situation résidentielle instable (115, CADA, squat). L' " aller vers » consiste donc en partie à « aller sur » les espaces que fréquentent les populations-cibles tout en prenant soin de réduire la distance sociale entre professionnels et patients. En effet, ce type d'intervention est non seulement fait pour aller chercher les personnes là où elles sont, mais aussi pour y créer ce que D. Mellier appelle des « opportunités interactionnelles " ${ }^{6}$ (2006), une notion là encore surtout 
utilisée et mobilisée dans le champ du travail social et de la psychologie pour décrire les registres d'action des professionnels travaillant auprès de personnes rencontrant des difficultés très complexes au quotidien (tels les personnes vivant à la rue) et avec lesquelles les logiques d'interactions doivent être spécifiques. Un professionnel de la santé qui décide d'aller faire des visites auprès de patients dans la rue ou dans un squat crée des "opportunités interactionnelles » non seulement parce qu'il va au-devant des patients dans leurs lieux de vie, mais aussi parce que sa présence ici, inhabituelle, n'est pas identifiée de la même façon que dans un lieu de soin et permet parfois lever des verrous dans les échanges patient-soignant. Bien sûr, tout ceci relève surtout du tâtonnement, de l'intuition du bricolage - au sens anthropologique du terme - et ne répond pas à des règles professionnelles explicites.

Cependant, les professionnels qui mettent en œuvre de telles actions se trouvent confrontés à un problème de fond : impulser une démarche d' " aller vers » entérine une vision « différentialiste » des publics et de l'accès aux droits. En effet, mettre en place et négocier des canaux spéciaux pour favoriser une catégorie de population parmi d'autres peut être vu comme contrevenant aux requis de l'universalisme. C'est un argument souvent manié par les financeurs en santé publique, par les politiques publiques et par les professionnels de santé, dont l'éthique repose sur l'égalité stricte entre toutes les personnes. Cette démarche, parce qu'elle implique de devoir formuler des critères de différentiation entre différentes populations, qui plus est, ici, des différenciations sur des critères d'origine nationale et de statut vis-à-vis du droit au séjour, implique la mobilisation d'arguments forts pour légitimer une action spécifique dans le domaine de la santé. Très souvent, c'est l'argument du risque à la santé publique qui est invoqué, cependant celui-ci n'est pas satisfaisant dans la mesure où il sous-entend que les migrants primo-arrivants feraient courir un risque sanitaire à la population générale. Un autre argument entendu sur le terrain est que la mise en place d'actions spécifiques à destination des migrants impliquerait un processus d'exclusion $\mathrm{du}$ domaine $\mathrm{du}$ droit commun. Ces raisonnements sont souvent opposés aux professionnels qui s'inscrivent dans les actions décrites plus haut, mais ceux-ci ne les retiennent généralement pas dans la mesure où ce sont aussi de parfaits arguments... pour ne rien faire. Nous avons pu constater sur le terrain que, pour maintenir et légitimer leurs actions, c'est le caractère novateur, multi-partenarial et dynamique qui est mis en avant.

C'est ainsi que les professionnels investissent les interstices sociaux et spatiaux de la précarité, c'est-à-dire qu'ils font une démarche vers des lieux et mettent en place des pratiques de l'entre-deux (entre la médecine et le travail social, entre le lieu de soin, la rue et le chez-soi). De cette façon, ils se trouvent dans un équilibre, précaire, consistant à mettre au point des actions spécifiques et mobiles tout en respectant le cadre commun de leur pratique professionnelle. Ni travailleurs sociaux, ni médecins généralistes classiques, ils se déplacent donc «avec un cadre dans la tête » (Mellier, 2006) dans des espaces interstitiels dans lesquels ils mettent en place des actions, temporaires mais répétées, sans que ces actions n'aient de statut particulier dans la pratique " orthodoxe » de la médecine. Nous entendons par là que les médecins que nous avons rencontré sortent des lieux communs de la médecine (le cabinet médical, l'hôpital) pour investir des espaces où l'on rencontre moins les médecins (mais plus généralement les travailleurs sociaux), non pas pour y faire uniquement de la médecine, mais pour saisir des opportunités de rencontrer des personnes qui seraient 
en marge du système de soin, dans l'optique d'anticiper les risques accrus et dans celui de les faire venir dans les lieux de soins :

Extrait d'entretien réalisé en juillet 2013 auprès d'un médecin généraliste exerçant dans un centre médical.

\section{Où et comment allez-vous voir les patients?}

En fait, il y a deux types de personnes que l'on voit. Il y a les demandeurs d'asile, les classiques, qui nous sont envoyés soit par une association, soit par la PADA ${ }^{7}$, soit par la plateforme MIE ${ }^{8}$... Peu importe, mais ceux-là on les voit. Il y a des demandeurs d'asile qu'on ne verra jamais parce qu'ils sont en squat. Et puis il y a surtout les autres, ceux qui ne sont pas passés par la PADA, qui sont en situation irrégulière. Et ceux-là, si on ne va pas les chercher, on ne les verra pas. En plus ils ont la trouille. D'où l'intérêt d'aller en squat: c'est d'aller à la pêche aux gens qu'on ne voit pas. Alors c'est vrai que quand on va dans les squats, il y a des gens que je connais, que j'ai déjà vu en consult'. C'est pas pour ceux-là que j'y vais. C'est pour ceux qu'on ne voit pas.

Mais ceux que vous connaissez peuvent vous aider à entrer en contact?

Tout à fait. C'est fait pour ça la démarche, pour aller vers les gens qui ne viennent pas. C'est la pêche, quoi. C'est vraiment la pêche.

Et vous devenez mobile?...

C'est indispensable. La médecine ça ne s'est jamais fait le cul sur une chaise. Enfin pas pour moi.

Mais ça implique de mettre en place une équipe mobile?

Oui, mais c'est une mobilité transitoire. On ne peut pas faire de la médecine dans un squat. Moi, je considère que c'est difficile. On peut en faire, mais c'est plus du bidouillage. Ce qui serait intéressant c'est de voir si les gens dans les squats on peut les amener ici, parce qu'ici on a le matériel, on a les interprètes. (..). Donc le but du jeu c'est de prendre ces gens-là, de les amener ici, en leur prévoyant un RDV, de façon organisée, pour qu'il y ait un médecin, un travailleur social, une infirmière, une interprète qui soient là au moment où ils se pointent.

"L'aller vers » revêt donc deux lectures. Cette pratique professionnelle peut être vécue comme subie, dessinant en creux le désinvestissement de l'État dans l'aide aux migrants et sa capacité à les intégrer dans le système de droit commun, et l'impérieuse nécessité à investir de nouvelles pratiques. Mais cette pratique peut aussi être vécue comme un choix qui rend le professionnel libre par rapport à son institution et lui permet de créer, adapter, innover dans son champ professionnel, en sortant des cadres figés de la pratique médicale.

Nous sommes là en présence de professionnels qui, à titre individuel, ont fait le choix de s'autosaisir pour «aller vers » les personnes. Ils se retrouvent en décalage avec leur institution car cette posture est finalement assez rare dans la profession (dans une ville comme Rennes, une quinzaine de médecins sont dans cette démarche). Cependant, ils composent avec les cadres institutionnels et, au final, ils pratiquent ce qui pour eux est le cœur de leur métier, tout en effectuant des actes professionnels singuliers.

Enfin, l'« aller vers » concerne les publics précaires, mais consiste aussi en des actions précaires. Ces actions sont fragiles, car elles sont constamment menacées financièrement (il faut mener des négociations âpres pour les légitimer et les faire 
financer), elles ne peuvent être pérennes et doivent se renouveler (sinon, elles ne pourraient pas être légitimées dans un contexte universaliste), et elles imposent une relation à l'autre qui risque de l'essouffler (en effectuant un travail incessant de réajustement, les actions peuvent détourner l'intérêt des personnes).

L'« aller vers » amène à des réflexions de fond complexes (la légitimité à agir, tout en respectant un cadre légal) et ouvre sur des réflexions théoriques qui touchent à la question des mobilités (des professionnels et des patients) dans des secteurs de l'action publique où les principes sont soumis à des impératifs de territorialisation, notamment à l'échelle de la ville et de ses quartiers.

\section{la ville et ses quartiers : ressource ou contrainte?}

Les travaux menés sur les trajectoires de soins des populations migrantes en France et sur les actions des professionnels travaillant à leur contact amènent à s'interroger sur les interfaces entre mobilités et territorialisation des questions de santé. Devant les impératifs de territorialisation des soins, notamment à l'échelle de la ville (et ses quartiers) ou de la région, les professionnels qui travaillent auprès de populations migrantes doivent s'organiser à la jonction entre mobilités (des personnes) et territoires (de l'action publique). L'échelon qu'ils favorisent pour mettre en œuvre leurs actions est celui du quartier, car c'est à cette échelle que l'attention des pouvoirs publics à destination des publics « différents » ou des actions « innovantes » est la plus facilement mobilisable. Cela tient, bien sûr, au fait que la municipalité ait décidé de se saisir des questions de santé dans sa politique d'action locale, et au fait que la ligne politique soit ouverte aux actions en direction des populations migrantes et de leurs difficultés sur son territoire.

D'un côté, le quartier peut être vu comme une ressource, dans la mesure où toute action publique territorialisée à cette échelle est un argument pour mobiliser rapidement des financements. Les villes sont des financeurs possibles, mais, dans ce cas, le service interlocuteur privilégié est souvent celui de la lutte contre les discriminations plus que celui de la santé publique. C'est aussi l'échelle la plus utilisée et la mieux maniée par tous les partenaires santé/social et société civile (associations de migrants, par exemple) : c'est à l'échelle du quartier que l'on peut légitimer une demande pour émarger à l'utilisation d'équipements et que l'on trouve les possibilités de mettre en œuvre des actions interdisciplinaires.

Mais, d'un autre côté, le quartier peut aussi être une échelle problématique, notamment en raison des risques de stigmatisation pour les personnes comme pour les professionnels. En effet, résider dans un quartier lorsque l'on est en situation précaire et devoir fréquenter des lieux de soins spécifiques, comme un centre de soins pour migrants ou une structure qui propose des consultations pour publics précaires, est lourd de sens pour les personnes, qui peuvent alors choisir de ne pas recourir aux soins. Parallèlement, la présence de centres de soins ou de programmes spécifiquement dédiés aux migrants est également potentiellement stigmatisant pour les acteurs du soin. Ainsi, au sein d'un quartier, un centre de soins pour migrants peut peiner à coordonner ses actions avec les autres acteurs locaux : l'étiquette "migrants malades » est généralement malvenue dans les actions d'une association visant à promouvoir l'intégration de migrants dans le quartier. Par conséquent, à cette échelle, on trouve 
des problèmes de communication entre acteurs, qui ne ciblent pas les mêmes catégories de migrants et n'ont pas la même vision de leurs actions de terrain :

Extrait d'entretien réalisé en juillet 2013 auprès d'un médecin généraliste exerçant dans un centre médical (NB: il s'agit d'un centre qui a déménagé ses locaux quelques mois avant l'entretien dans un autre quartier de la ville).

Quels étaient vos liens avec le centre de soins du quartier (ancienne localisation)?

Alors ça on n'y va plus depuis qu'on a quitté le quartier. (...). On y allait très peu, on y allait une fois ou deux par an. Enfin moi j'y suis jamais allé, ce devait être la secrétaire qui y allait.

Et vous alliez voir des patients? Vous adressiez des patients?

Non, on y allait plus parce qu'il y a une population migrante importante dans le quartier (mais pas dans le cadre des primo-arrivants, dans le cadre des vieux migrants). Je pense qu'il y avait des échanges autour de ça. Bon, moi je ne connais pas. Mais ces structures là, bon, il y a plein de comités, de machins, de bidules, tout ça qui sont en place, à mon avis c'est... j'avoue que j'ai un peu de mal dans ce genre de structure.

\section{Et dans ce quartier-là (nouvelle localisation)?}

Dans ce quartier-là, c'est vrai qu'on n'y est pas depuis très longtemps. Ça ne fait jamais que deux mois qu'on est là, et on n'a jamais trop prospecté.

Ces problèmes de communication et ces difficultés à travailler en coordination à l'échelle du quartier proviennent aussi des contours politiques de ces territoires. En effet, les actions mises en place à destination des migrants sont souvent délimités par limites du CUCS qui définit un certain nombre de champs d'action considérés comme "prioritaires", et attribue une partie de la mise en œuvre de ces actions aux professionnels travaillant dans le quartier. Malgré tout, le quartier reste un des échelons d'intervention le plus souple, car les interlocuteurs sont plus faciles à mobiliser (élus, services municipaux): c'est l'échelon dans lequel peuvent être inventées et ajustées les actions les plus diverses (ASV/RVH), mais c'est aussi l'échelon duquel on peut s'extraire pour aller voir les populations là où elles « résident ».

\section{Conclusion}

31 L'organisation des soins à destination des migrants relève de compositions et de négociations entre acteurs concernés, qui, le plus souvent, devant les situations d'urgence, s'autosaisissent de la question. Les actions qui sont alors mises en place s'organisent en fonction des forces en présence, dans les territoires de la ville et éventuellement des régions. Elles ont pour caractéristiques d'être « inventives " pour rester au plus près des populations, ce qui va de pair avec une volonté des professionnels à s'adapter aux patients, tout en respectant au plus près les demandes des institutions et des logiques professionnelles. Cependant, les modes de territorialisation des actions qui ont été décrites dans cet article (actions inscrites dans les «espaces interstitiels») peut induire différentes difficultés: cela implique de jongler avec différentes modalités de différentiation entre les différents publics, de s'attaquer de front à la question de la stigmatisation (des publics/des acteurs), de prendre en compte et régler, si possible, les problèmes de communication entre les structures. Mais cela souligne aussi le foisonnement de modes d'actions innovants 
construits à la marge du système de santé tout en respectant le cœur des actions à destination des publics concernés. Les notions et catégories en lien avec la territorialisation de l'action publique doivent donc être examinées plus attentivement dans le cas des migrants primo-arrivants. En effet, les territoires de la santé oscillent entre paradoxes et ambiguïtés de la contrainte institutionnelle (nationale/locale) et des situations locales très spécifiques (des publics/des acteurs et de leurs modes de mobilisation).

Il s'avère nécessaire de pendre en compte en même temps le point de vue des acteurs de la santé et du social dont les actions oscillent entre l'injonction d'implantation d'action dans des quartiers (avec la volonté de «capter» les populations des actionscibles), et l'impératif, pour certains, de se mobiliser pour inventer des pratiques pour trouver et soigner les « invisibles ». En parallèle, dans la mesure où la santé n'est pas qu'une question d'accès aux soins, il s'agit également de pendre en compte les réalités quotidiennes des migrants, constituées de mobilités contraintes. Celles-ci ne sont pas corollaires d'une exclusion totale ou d'une absence totale de liens, dans la mesure où des pratiques d'«aller vers" mises en place par les professionnels peuvent faire ressource. Et, de la même façon, parmi les personnes que nous avons interrogé, même celles qui subissent les effets délétères des trajectoires résidentielles instables (les personnes en CADA, dépendantes du 115 et/ou en hébergement provisoire chez des proches) peuvent développer une connaissance de la ville en mobilisant des «savoircirculer». D'un point de vue théorique, cela nous rapporte aux études sur les différentes formes d'inscription spatiale des actions publiques en santé, et les pratiques des populations et des professionnels, qui, à l'épreuve de l'échelle micro-locale, ne peuvent être étudiées à l'aune de catégories figées (Dureau et Hily 2009; Ramadier et Depeau 2011).

\section{BIBLIOGRAPHIE}

Beauchemin, C., Hamel, C., et Simon, P. (2010). Enquête « Trajectoires et Origines : la diversité des populations en France ", Premiers résultats . 168. Document de travail de l'Ined. INED.

Bergeon C. (2014). « Habiter l'éphémère. Le squat comme lieu de vie et comme ressource dans les parcours de migrants internationaux ». ESO Travaux et Documents , ${ }^{\circ}$ 37. http://eso.cnrs.fr/ TELECHARGEMENTS/revue/ESO_37/1bergeon.pdf .

Carde, E. (2006). « On ne laisse mourir personne ». Les discriminations dans l'accès aux soins. Travailler , 16, 5780 .

Carde, E. (2007). Les discriminations selon l'origine dans l'accès aux soins. Santé Publique , 2 , 99 109.

Chauvin, P., Parizot, I. (2005). Santé et recours aux soins des populations vulnérables . Paris : INSERM.

Chauvin, P., Simonnot N., Douay C., et Vanbiervliet F. (2014). Access to healthcare for people facing multiple vulnerability factors in 27 cities across 10 countries . Report on the social and medical data 
gathered in 2013 in eight European countries, Turkey and Canada. Paris: International Network of Médecins du Monde.

Cognet, M., \& Hamel, C. (2012). Santé des migrants en France : l'effet des discriminations liées à l'origine et au sexe. Revue Européenne des Migrations Internationales , 28 (2).

Cognet, M., Gabarro C., Adam-Vezina, E. (2009). « Entre droit aux soins et qualité des soins ». Hommes et Migrations , $\mathrm{n}{ }^{\circ}$ 1282. http://www.hommes-et-migrations.fr/index.php?/numeros/ sante-et-droits-des-etranger-realites-et-enjeux/5725-entre-droit-aux-soins-et-qualite-des-soins .

Cognet, M., Montgomery C. (2007). Ethique de l'altérité. La question de la culture dans le champ de la santé et des services sociaux. Presses de l'Université Laval. Québec.

Cognet, Marguerite, Anne-Cécile Hoyez, et Christian Poiret. 2012. « Expériences de la santé et du soin en migration: entre inégalités et discriminations ». Revue Européenne des Migrations Internationales $28(2): 710$.

De Jonckheere, C., Chalverat C., Rufini Steck L., Elghezouani A. 2011. Le thérapeute et le diplomate. Modélisation de pratiques de soin aux migrants . CERES. Genève : IES. http://lectures.revues.org/ 6945 .

Demazière, D., \& Samuel, O. (2010). Inscrire les parcours individuels dans leurs contextes. Temporalités. Revue de sciences sociales et humaines , (11). Consulté à l'adresse http:// temporalites.revues.org/1167?lang=en

Dureau, F., \& Hily, M.-A. (2009). Les mondes de la mobilité . Rennes : Presses Universitaires de Rennes.

Fassin, D., éd. (1998). Les figures urbaines de la santé publique : enquête sur des expériences locales . 1 vol. Recherches (Paris. 1994), ISSN 1258-4002. Paris, France : Éd. la Découverte.

Fleuret, S, et Séchet R. (2006). «Spatialité des enjeux de pouvoir et des inégalités : pour une géographie sociale de la santé ». In Penser et faire la géographie sociale. Contributions à une épistémologie de la géographie sociale, Raymonde Séchet et Vincent Veschambre, Presses Universitaires de Rennes, 400 p. Rennes.

Hassenteufel, P., Le Bihan-Youinou B., Loncle P., et Vion A. (1998). «L'émergence problématique d'une nouvelle santé publique. Forums d'action publique et coalitions de projets à Rennes et à Brest ». In Figures urbaines de la santé publique. Enquête sur les expériences locales, Didier Fassin, La Découverte. Paris.

Hoyez, A.-C. (2011). « L'accès aux soins des migrants en France et la "culture de l'initiative locale". Une analyse des contextes locaux à l'épreuve des orientations nationales ». Cybergeo : European Journal of Geography , Espace, Société, Territoire. http://cybergeo.revues.org/24796 .

Hoyez, A.-C. (2012). « Mobilités et accès aux soins des migrants en France ». Géoconfluences . http://geoconfluences.ens-lyon.fr/doc/transv/sante/SanteScient4.htm .

Izambert, C. (2010). 30 ans de régressions dans l'accès aux soins. Plein Droit , (86). Consulté à l'adresse http://www.gisti.org/spip.php?article2102

Lelièvre, É., Courgeau D. (1996). « Changement de paradigme en démographie ». Population 51 (3): 645 54. doi:10.2307/1534487.

Ma Mung, E. (1999). La dispersion comme ressource. Cultures \& Conflits , (33-34). Consulté à l'adresse http://conflits.revues.org/index225.html

Maille, D., \& Veïsse, A. (2000). Exclus des soins à l'heure de la CMU : comment réintégrer le système de santé ? La revue du praticien - Médecine Générale , (502). 
Marche, H., Pian A. (2015). «Figures de l'altérité et de l'indicibilité dans les soins oncologiques ». In Les malentendus culturels dans le domaine de la santé, Pascal Hintermeyer, David Le Breton, et Gilles Profita. Presses Universitaires de Strasbourg.

Médecins du Monde. (2009). L'accès aux soins : un droit non respecté en Europe (Médecins du Monde).

Mellier, D. (2006). Précarité psychique et dispositifs d'intervention clinique. Pratiques Psychologiques , 12(2), 145 155. doi:10.1016/j.prps.2006.01.008

Mizrahi, \& Mizrahi. (2000). Les étrangers dans les consultations des centres de soins gratuits. Hommes et Migrations . Consulté à l'adresse http://argses.free.fr/textes/ 18_Etrangers_dans_centres_de_soins_gratuits.pdf

Olivier de Sardan, J.-P. (1995). « La politique du terrain ». Enquête. Archives de la revue Enquête , $\mathrm{n}$ ० 1 (octobre) : 71 109. doi:10.4000/enquete.263.

Parizot, I. (2003). Soigner les exclus . Le Lien Social. Paris: PUF.

Pian, A. (2012). De l'accès aux soins aux «trajectoires du mourir ». Les étrangers atteints de cancer face aux contraintes administratives. Revue Européenne des Migrations Internationales , 2 .

Ramadier, T., \& Depeau, S. (2011). Se déplacer pour se situer : Places en jeu, enjeux de classes .PU Rennes.

Roche, P. (2007). Les défis de la proximité dans le champ professionnel. Nouvelle revue de psychosociologie , 3(1), 63 82. doi:10.3917/nrp.003.0063

Rode, A. (2009). « L'émergence du non-recours aux soins des populations précaires: entre droit aux soins et devoirs de soins ». Lien social et politique, Pauvreté, précarité: quels modes de régulation?, 61: 14958.

Rullac, S. (2014). La scientifisation du travail social. Politiques et interventions sociales. Presses de l'EHESP.

Strauss, A., Baszanger I. (1991). La trame de la negociation . Logiques sociales. Paris: L'Harmattan. The Franco-British Working Group on Migration, Health and Wellbeing. (2009). Health, Wellbeing and Immigrant Populations in France and Britain. International Journal of Migration, Health and Social Care , 5 (4), 4 19. doi:10.5042/ijmhsc.2010.0228

Thomas, F. (2010). Transnational health and treatment networks: Meaning, value and place in health seeking amongst southern African migrants in London. Health \& Place , 16(3), 606 612. doi: 16/j.healthplace.2010.01.006

Vallée, J., Frohlich, K., Kestens, Y., \& Shareck, M. (2014). Accès aux ressources et santé mentale à Montréal - Métropolitiques. Consulté 15 juillet 2014, à l'adresse http://www.metropolitiques.eu/ Acces-aux-ressources-et-sante.html

Vallée, J., Roux, G. L., Chaix, B., Kestens, Y., \& Chauvin, P. (2014). The 'constant size neighbourhood trap' in accessibility and health studies. Urban Studies , 0042098014528393. doi : $10.1177 / 0042098014528393$

Warin, P. (2013). « L'accès aux soins des populations vulnérables ». In Colloque "Accès aux soins des plus démunis" . Paris, France. https://halshs.archives-ouvertes.fr/halshs-00875330 .

Yeni, P. (2010). Prise en charge médicale des personnes infectées par le VIH . La Documentation Française. Paris: Ministère de la Santé et des Sports. http://www.sante.gouv.fr/IMG/pdf/ Rapport_2010_sur_la_prise_en_charge_medicale_des_personnes_infectees_par_le_VIH_sous_la_direction_du_Pr_Patrick_Yeni.pdf. 
Zeneidi, D., Fleuret S. 2007. « Fixes sans domicile, réflexion autour de la mobilité des SDF ». L'Espace géographique Tome 36 (1): 114.

\section{NOTES}

1. Cet article fait suite à des réflexions entamées lors de journées d'étude organisées par l'INED et le GIS-CIST sur la dimension locale des faits de santé en milieu urbain ( http://pole_suds.site.ined.fr/fr/les_journees/quartiers_sante/ ), organisé en septembre 2012 par Clémentine Rossier et Julie Vallée.

2. Projet MIGSAN «MIGRATIONS ET SANTÉ: Analyse des variations dans l'accès aux soins des populations migrantes à l'échelle locale » (financement Réseau National des MSH ; MSH Bretagne/MSHS Poitiers/Maison des Sciences de l'Homme Ange Guépin Nantes).

3. Centre d'Accueil des Demandeurs d'Asile

4. Le centre médical dispose d'interprètes en 15 langues.

5. Le rapport Yeni relatif à la prise en charge des personnes infectées par le VIH, remis à la Ministre de la Santé et des Sports en 2010, inclut un chapitre relatif "aux conditions de vie pour un succès thérapeutique " qui insiste précisément sur le logement et l'hébergement.

6. Cette notion est peu développée en sciences humaines et sociales; elle se rencontre dans le champ des sciences de la communication (où elle évoque les possibilités de changements dans une activité particulière, comme la conversation) et dans le champ de la sociolinguistique (où elle se rapporte à un mécanisme d'apprentissage qui permet l'acquisition d'une langue étrangère).

7. Plateforme d'Accueil des Demandeurs d'Asile

8. Mineurs Isolés Etrangers

\section{RÉSUMÉS}

Cet article aborde les enjeux de santé relatifs aux populations migrantes primo-arrivantes en les interrogeant du point de vue de l'expérience des individus et des professionnels qui travaillent à leur contact. D'un côté, il interroge les déterminants sociaux de la santé et de l'accès aux soins tels qu'ils se présentant pour les migrants, en prenant en compte l'ensemble des contraintes, des ruptures, mais aussi des ressources mobilisables pour la santé et les soins. D'un autre côté, l'article interroge la façon dont les professionnels de la santé mettent en place des actions à l'égard des migrants, dans un contexte institutionnel national et local changeant: comment mettre en place des actions à destination de ces publics? Comment faire en sorte que les organismes et structures existantes s'adaptent aux situations des populations les plus susceptibles de rester en marge du système de santé ? 
This article deals with health and healthcare access of migrant populations, placing the debate around the point of view of migrants (individuals and family) as well as of professionals working with them. The article questions on the one hand the social determinants of health and the determinants of healthcare access by taking into consideration the set of constraints, incoherencies and resources available for health and healthcare. On the other hand, it shows how healthcare professionals implement new forms of action destined to migrants. In a changing local and national institutional context the issue raised is how to set up actions towards migrants in the domain of health and healthcare. Further, it considers how to ensure that existing structures and organizations are adapted to the situation of a population that is, more than others, likely to remain at the margins of the healthcare system? The article studies the administrative and juridical healthcare access for migrants in France. Two main assessments can be put forward: first of all, the migrants' pathways to healthcare are characterized by long delays before accessing primary care; second, healthcare pathways appear as "coordinated" (between emergency healthcare and common access) only when residential and administrative situations are solved and stable. Our global observations are based on fieldwork in France; they show that there are very heterogeneous ways to access healthcare for newly arrived migrants, and generally that they stay longer than other precarious populations in the circuit of emergency humanitarian health. We also notice that the difficulties in accessing healthcare for migrants are only a part of the difficulties experienced in migratory experience. We attempt to describe types of daily barriers (administrative situations, difficulties in accessing rights, negative representations of migrants within society and/or social and sanitary services, language) and to underline the necessity of taking into account the socio-spatial dimensions of healthcare access in everyday life. The article includes a methodological section explaining the fieldwork (interviews with patients and professionals) and describing the content of the interview grid (see figure 1.) The patients' points of view are described through the use of "synthetic biographies". These biographies underline the everyday spatial constraint obliging individuals to develop tactics and strategies to move to the city (and between cities) in order to access different services. The professionals' points of view are analyzed in regard to their professional practices, and the way they use some "tinkering" to favor new forms of actions targeting migrants. We can observe in the fieldwork the multiplication of "go towards" practices, usually used in the medicosocial field (and generally to set up actions meant to "go towards" individuals identified as precarious -in the domain of addictions or HIV-AIDS). These actions include a spatial register: actions tend to "go towards" people living in the street or in squats. These professional practices lead to a type of medicine that is institutionally framed but concern very specific professional acts. We thus analyze the way this tinkering becomes possible and how it appears in the interviews we have conducted with general practitioners. The discussion and conclusion aim at considering the different spatial inscriptions of these forms of actions in public health. We have also studied the situation of migrants in precarious situations: on the one hand they endure the deleterious effects of their living conditions, but they can also develop very original spatial knowledge and multi-situated sociability that can be analyzed as "“"mobility know-how”.

\section{INDEX}

Mots-clés : migrants, accès aux soins, mobilités, pratiques professionnelles, expérience de la santé et du soin

Keywords : migrants, healthcare access, mobility, professional practices, health and healthcare experience 
AUTEUR

\section{ANNE-CÉCILE HOYEZ}

Chargée de recherche, CNRS UMR ESO, Université Rennes 2 